\title{
Association Study of Two Polymorphisms in Vitamin D Pathway with Multiple Sclerosis in the Moroccan Population
}

\author{
Asmae Skalli1, El Hachmia Ait Ben Haddou1,2, Rachid Tazi-Ahnini ${ }^{3}$, Ghislain Armel Mpandzou', \\ Houyam Tibar',2, Naima Bouslam², Ali Benomar',2, Khadija Hajjout ${ }^{4}$, Mohamed Yahyaoui ${ }^{1,2}$, \\ Elmostafa El Fahimes, Ahmed Bouhouche ${ }^{1,2}$
}

\author{
${ }^{1}$ Research Team in Neurology and Neurogenetics, Medical School and Pharmacy, Mohammed V University, Rabat, Morocco \\ ${ }^{2}$ Department of Neurology and Neurogenetics, Specialties Hospital, Rabat, Morocco \\ ${ }^{3}$ Laboratory of Biotechnology, Medical School and Pharmacy, Mohammed V University, Rabat, Morocco \\ ${ }^{4}$ National Blood Transfusion Center, Rabat, Morocco \\ ${ }^{5}$ Assistance Units for Scientific and Technical Research, Rabat, Morocco \\ Email: asmae-skalli@hotmail.fr
}

How to cite this paper: Skalli, A., Ait Ben Haddou, E., Tazi-Ahnini, R., Mpandzou, G.A., Tibar, H., Bouslam, N., Benomar, A., Hajjout, K., Yahyaoui, M., El Fahime, E. and Bouhouche, A. (2017) Association Study of Two Polymorphisms in Vitamin D Pathway with Multiple Sclerosis in the Moroccan Population. World Journal of Neuroscience, 7, 363-375. https://doi.org/10.4236/wjns.2017.74028

Received: September 11, 2017

Accepted: October 21, 2017

Published: October 24, 2017

Copyright ( 2017 by authors and Scientific Research Publishing Inc. This work is licensed under the Creative Commons Attribution International License (CC BY 4.0).

http://creativecommons.org/licenses/by/4.0/

(c) (i) Open Access

\begin{abstract}
Background: Hypovitaminosis D is reported through the literature to be involved in autoimmune diseases such as multiple sclerosis (MS). In the last decade, numerous studies have investigated the association of single nucleotide polymorphisms (SNPs) with MS, including rs2248359 (CYP24A1) and rs703842 (CYP27B1) that are involved in vitamin D metabolic pathway. However, results were conflicting, probably due to ethnic differences between the studied populations. In this context, the present study aimed to analyze the association between these two SNPs and MS within the Moroccan population. Methods: rs2248359 and rs703842 were genotyped in 113 patients and 146 healthy controls. To assess their association with the disease risk, we compared the genotypic and allelic frequencies between the study groups. We also explored their possible influence on certain clinical features (age at onset, type, disability status and severity score) and with vitamin D3 serum level (DSL) by comparing mean values of these variables between the different genotypes. Results: No statistically significant differences in the distribution of both SNPs were found between patients and controls. A trend has emerged concerning the minor $\mathrm{G}$ allele of rs703842 which appears to have a protective effect on developing MS, but this result remained slightly below significance. Also, the two polymorphisms had no impact on the clinical features tested and the DSL. Conclusion: There is no convincing evidence that rs2248359 and rs703842 are associated with MS risk, its clinical features or vitamin D level in Moroc-
\end{abstract}


cans. Further larger investigations are needed to confirm these findings.

\section{Keywords}

Multiple Sclerosis Risk, rs2248359, rs703842, Clinical Features, Serum $25(\mathrm{OH}) \mathrm{D}_{3}$ Level

\section{Introduction}

Multiple sclerosis (MS) is a chronic autoimmune disease of the central nervous system and the most common non-traumatic cause of neurologic disability in young and middle-aged adults [1]. It is characterized by a complex pathogenesis in which demyelinating lesions, axonal degeneration and gradual accumulation of sclerotic plaques are mainly responsible for this handicap [2]. Both environmental risk factors and genetic susceptibility contribute to MS etiology [3] [4].

Among the known environmental risk factors, low concentration of vitamin D in blood circulation seems to have a significant role on MS pathogenesis, probably due to the intervention of vitamin $\mathrm{D}$ in several pathways of the immune system [5]. Indeed, besides its fundamental role in calcium homeostasis and bone metabolism, vitamin D also has numerous additional functions, especially anti-inflammatory and immunomodulatory actions which could be involved in autoimmune diseases like MS. These include inhibition of $\mathrm{B}$ and $\mathrm{T}$ cells proliferation and differentiation, regulation of antigen presenting cells differentiation and conversion of cytokine environment from a pro-inflammatory state (Th1/Th17-cell-mediated) to a rather anti-inflammatory one (Th2-cell-mediated). Furthermore, calcitriol $\left(1.25(\mathrm{OH})_{2} \mathrm{D}\right)$, which is the active metabolite of vitamin $\mathrm{D}$, seems to have potential actions within central nervous system cells, such as neuronal functioning, neuroprotection and myelination. Resulting from this, the vitamin D status likely contributes to the risk of developing MS, and could also influence the main clinical variables of MS patients after the disease onset [6] [7].

Concerning the genetic susceptibility of MS, haplotypes in the major histocompatibility complex (MHC), particularly HLA DRB1*1501-DQB1*0602 (HLA-DR15), are the risk factors exerting the strongest effect [4]. But over the last decade, numerous genome-wide association studies (GWASs) have reported more than 50 non-MHC loci involved in the MS predisposition. It was therefore suggested that the MS susceptibility profile consisted of many risk alleles, each contributing a relatively small effect to the overall risk [8]-[13]. Among these, single nucleotide polymorphisms (SNPs) of vitamin D metabolizing genes, including rs2248359 and rs703842, have been found to be implicated in MS pathogenicity in Caucasian populations [12] [13]. rs703842 is located in the 5' region of the CYP27B1 gene (12q13.1-q13.3) which encodes a 1- $\alpha$-hydroxylase enzyme (cytochrome P450, family 27 , subfamily B, peptide 1 ) that catalyzes the conversion of $25(\mathrm{OH}) \mathrm{D}$ to $1.25(\mathrm{OH})_{2} \mathrm{D} 3$, 
the physiologically active form of vitamin D. Similarly, rs2248359 is located in the 5' region of the CYP24A1 gene (20q13) which encodes a 24-hydroxylase enzyme (cytochrome P450, family 24, subfamily A, peptide 1) that initiates deactivation and degradation of both $25(\mathrm{OH}) \mathrm{D}$ and $1.25(\mathrm{OH})_{2} \mathrm{D} 3$. Because of their location in genomic regions predicted to be promoters for their neighboring genes, both SNPs might change the expression of these genes, and thus contribute to tissue availability of active vitamin D3. Thereby, they might modify the vitamin D influence on immune function, which could in turn affect susceptibility to MS [14] [15] [16].

Many candidate gene studies carried out in different countries have attempted to replicate the association between the two SNPs and MS but results are conflicting, possibly due to differences in ethnic origins of the concerned participants. The present study aimed to analyze the genetic association of rs2248359 and rs703842 with MS disease in Moroccan population, and to explore their possible interactions with certain clinical features (age at onset, MS type, disability status and severity score) as well as with the vitamin D3 serum level (DSL).

\section{Materials and Methods}

\subsection{Case-Control Selection}

One hundred thirteen Moroccan MS patients were recruited through the neurological department of the Specialties Hospital (Rabat, Morocco) between January 2012 and March 2015. Some general and clinical information were collected at the time of blood sampling, such as sex, age, ethnical origins, MS type, age at onset and duration of the disease. The disability was evaluated using the Expanded Disability Status Scale (EDSS). All patients met the 2010 McDonald's criteria; 50 of them showed relapsing remitting (RRMS), 40 secondary progressive (SPMS) and 23 primary progressive (PPMS) MS type. The disease course was divided into two subcategories, PPMS and RRMS-SPMS since SPMS patients were first RRMS ones. The age at onset of the disease corresponds to self-reported patient's age at the apparition of first symptoms. To assess the disease severity, the EDSS score and the disease duration were used to calculate the Multiple Sclerosis Severity Score (MSSS) by referring to the global MSSS table [17].

One hundred forty six sex and age matched healthy subjects served as controls. They were all blood donors recruited in the National Blood Transfusion Center (Rabat, Morocco) from November 2013 to March 2014. Only subjects with no personal or familial history of inflammatory and autoimmune disease (MS, diabetes, psoriasis, eczema, cancer, arthritis...) and who have not followed a potentially mutagenic treatment (chemotherapy, radiotherapy...) were enrolled.

Furthermore, no patient or control having a foreign ancestor has been retained, all were of Moroccan origin. Besides, measurement of DSLs was carried out in a previous study involving the same subjects (in press). All participants provided written informed consent and the study protocol was approved by the Ethics Committee for Biomedical Research (Rabat, Morocco). 


\subsection{Blood Collection and DNA Extraction}

For both patients and controls, blood was drawn into a $6 \mathrm{~mL}$ EDTA-containing vacutainer tubes. After lysis of red cells, leucocyte pellets obtained were stored at $-25^{\circ} \mathrm{C}$. DNA extraction was carried out using Isolate II genomic DNA Kit from Bioline, and the DNA quantity and quality was determined by the Nanodrop 8000 Spectrophotometer from Thermo Scientific. The DNA samples were finally stored at $-20^{\circ} \mathrm{C}$ until use.

\subsection{SNP Genotyping}

Two SNPs, rs2248359 and rs703842 respectively located near CYP24A1 and CYP27B1 genes, were selected for their role in the vitamin D metabolism and their potential association with MS based on previously published genome-wide and candidate-gene studies.

Genotyping of rs2248359 (C > T) was performed with the PCR-RFLP method. The following primers were designed with Primer3 v4.0 software and used for PCR: forward 5'-AGTTAGGAAATGCGCCTTGAG-3' and reverse 5'-GGATCAGGTTGAAAGGATTCG-3'. The 326 bp PCR-generated amplicons were systematically checked by electrophoresis on $2 \%$ agarose gel. Restriction enzyme SacII (recognition site: CCGCGG) from New England Biolabs served for the amplicons' digestion. SacII recognizes the C allele of the CYP24A1 gene and cut to generate two fragments of $100 \mathrm{bp}$ and $226 \mathrm{bp}$ which segregate after electrophoresis on 3\% agarose gel. Three randomly selected samples of each genotype (namely $7 \%$ of the total sample) were subjected to regenotyping through DNA sequencing. Concordance rate for these quality-control samples was $100 \%$.

Rs703842 (A > G) has been genotyped using PCR followed by DNA sequencing. As previous, primers used for amplification were designed with Primer3 v4.0 software: forward 5'-GGGTAGTCATGAACACCAGT-3' and reverse 5'-GGGGAAGAAAGTTCTACGTAAT-3'. PCR products were purified with the ExoSAP-IT kit from Affymetrix, then sequenced using the reverse PCR primer as sequencing primer and the ABI Prism BigDye ${ }^{\circledast}$ v3.1 Cycle Sequence Kit on an ABI 3130xl Genetic Analyzer, both from Applied Biosystems, according to the manufacturer's protocol. Raw data were analyzed using Seqscape v2.5 software, also from Applied Biosystems.

Synthesizing of primers and sequencing were both performed at in the Functional Genomics Platform (PGF) of the National Center for Scientific and Technical Research (CNRST, Rabat, Morocco).

\subsection{Statistical Analysis}

Data were analyzed using the SPSS software 18 (v18.0, SPSS Inc., Chicago, IL). Descriptive statistics were used to assess the demographic variables, the disease characteristics and the DSL.

Prior to association tests, deviation from Hardy-Weinberg equilibrium (HWE) was examined in the control group using a chi-square $\left(\chi^{2}\right)$ test. Associations of 
rs2248359 and rs703842 with MS risk were assessed by comparing genotypic and allelic frequencies between patients and controls. Concerning genotypic frequencies, subjects were stratified according to three genetic models: additive, dominant (common allele homozygotes versus heterozygotes and minor allele homozygotes) and recessive (common allele homozygotes and heterozygotes versus minor allele homozygotes). Differences in the genotypic and allelic distribution between study groups were determined using the $\chi^{2}$ test for statistical significance. Odds ratios (ORs) with 95\% confidence intervals (CIs) were estimated for dominant, recessive and allelic models.

Four clinical characteristics (age at onset, MS type, EDSS score and MSSS) and DSL were also tested for association with both polymorphisms. Differences in mean values of these continuous variables between the different genotypes were evaluated using the univariate analysis of variance (ANOVA); except for MS type (categorical variable) which frequency analysis in regards to genotypes was performed using the contingency coefficient $(C)$. In all cases, $p$ values less than 0.05 (two-sided) were considered statistically significant.

\section{Results}

One hundred thirteen patients with MS and 146 unrelated healthy controls were enrolled. Detailed characteristics of the study population have been previously described (in press) and are summarized in Table 1.

Genotype frequencies for rs2248359 and rs703842 were in HWE among controls ( $\mathrm{p}=0.26$ and $\mathrm{p}=0.63$ respectively). The genotypic and allelic distribution of both polymorphisms in MS cases and controls are presented in Table 2. No

Table 1. Characteristics of MS patients and controls.

\begin{tabular}{|c|c|c|}
\hline & Patients $n=113$ & Controls $\mathrm{n}=146$ \\
\hline \multicolumn{3}{|l|}{ Gender: } \\
\hline Males $^{1}$ & $29(25.66)$ & $39(26.71)$ \\
\hline Females $^{1}$ & $84(74.34)$ & $107(73.29)$ \\
\hline Age (years) ${ }^{2}$ & $36.8 \pm 10.8(18-60)$ & $33.6 \pm 11.3(18-60)$ \\
\hline Age at onset (years) ${ }^{2}$ & $29.82 \pm 9.96$ & - \\
\hline Disease duration (years) ${ }^{3}$ & $5(2-8)$ & - \\
\hline \multicolumn{3}{|l|}{ MS type: } \\
\hline RRMS and SPMS ${ }^{1}$ & $90(79.65)$ & - \\
\hline PPMS $^{1}$ & $23(20.35)$ & - \\
\hline $\mathrm{EDSS}^{2}$ & $3.47 \pm 1.92$ & - \\
\hline MSSS $^{2}$ & $5.57 \pm 2.82$ & - \\
\hline $\mathrm{DSL}(\mathrm{ng} / \mathrm{ml})^{2}$ & $11.69 \pm 6.97$ & $12.98 \pm 6.58$ \\
\hline Hypovitaminosis $\mathrm{D}(\mathrm{DSL}<30 \mathrm{ng} / \mathrm{ml})^{1}$ & $110(97.35)$ & $144(98.63)$ \\
\hline
\end{tabular}

${ }^{1}$ Number and percentage $\mathrm{N}(\%)$; ${ }^{2}$ mean and standard deviation; ${ }^{3}$ median and interquartile range; MS: Multiple Sclerosis; RRMS: Relapsing-Remitting Multiple Sclerosis; SPMS: Secondary Progressive Multiple Sclerosis; PPMS: Primary Progressive Multiple Sclerosis; EDSS: Expanded Disability Status Scale; MSSS: Multiple Sclerosis Severity Scale; DSL: vitamin D3 Serum Level. 
Table 2. Comparison of genotypic and allelic distribution of rs2248359 (CYP24A1) and rs703842 (CYP27B1) polymorphisms between MS patients and controls.

\begin{tabular}{|c|c|c|c|c|c|c|c|}
\hline SNP (gene) & Genotype/All & & MS Patients $n=113(\%)$ & Controls $\mathrm{n}=146(\%)$ & $\mathrm{X}^{2}$ & $\mathrm{p}$ & OR (95\% CI) \\
\hline \multirow{9}{*}{$\begin{array}{l}\text { rs2248359 } \\
\text { (CYP24A1) }\end{array}$} & & $\mathrm{CC}$ & $28(24.78)$ & $44(30.14)$ & \multirow{3}{*}{1.38} & \multirow{3}{*}{0.50} & \\
\hline & \multirow[t]{2}{*}{ additive genetic model } & $\mathrm{CT}$ & $59(52.21)$ & $66(45.21)$ & & & \\
\hline & & TT & $26(23.01)$ & $36(24.66)$ & & & \\
\hline & \multirow{2}{*}{$\begin{array}{l}\text { dominant genetic } \\
\text { model }\end{array}$} & $\mathrm{CC}$ & $28(24.78)$ & $44(30.14)$ & \multirow{2}{*}{0.91} & \multirow{2}{*}{0.34} & \multirow{2}{*}{$0.76(0.44-1.33)$} \\
\hline & & $\mathrm{CT}+\mathrm{TT}$ & $85(75.22)$ & $102(69.86)$ & & & \\
\hline & \multirow{2}{*}{$\begin{array}{l}\text { recessive genetic } \\
\text { model }\end{array}$} & $\mathrm{CC}+\mathrm{CT}$ & $87(76.99)$ & $110(75.34)$ & \multirow{2}{*}{0.10} & \multirow{2}{*}{0.76} & \multirow{2}{*}{$0.91(0.51-1.63)$} \\
\hline & & $\mathrm{TT}$ & $26(23.01)$ & $36(24.66)$ & & & \\
\hline & \multirow{2}{*}{ allelic model } & $\mathrm{C}$ & $115(50.88)$ & $154(52.74)$ & \multirow[b]{2}{*}{0.18} & \multirow[b]{2}{*}{0.67} & \multirow[b]{2}{*}{$0.93(0.65-1.31)$} \\
\hline & & $\mathrm{T}$ & $111(49.12)$ & $138(47.26)$ & & & \\
\hline \multirow{9}{*}{$\begin{array}{c}\text { rs703842 } \\
(\mathrm{CYP} 27 \mathrm{~B} 1)\end{array}$} & \multirow{3}{*}{ additive genetic model } & AA & $82(72.57)$ & $90(61.64)$ & \multirow{3}{*}{3.46} & \multirow{3}{*}{0.18} & \\
\hline & & AG & $26(23.01)$ & $48(32.88)$ & & & \\
\hline & & GG & $5(4.42)$ & $8(5.48)$ & & & \\
\hline & dominant genetic & $\mathrm{AA}$ & $82(72.57)$ & $90(61.64)$ & \multirow{2}{*}{3.41} & \multirow{2}{*}{0.07} & \multirow{2}{*}{$1.65(0.97-2.80)$} \\
\hline & model & $\mathrm{AG}+\mathrm{GG}$ & $31(27.43)$ & $56(38.36)$ & & & \\
\hline & \multirow{2}{*}{$\begin{array}{l}\text { recessive genetic } \\
\text { model }\end{array}$} & $\mathrm{AA}+\mathrm{AG}$ & $108(95.58)$ & $138(94.52)$ & \multirow{2}{*}{0.15} & \multirow{2}{*}{0.70} & \multirow{2}{*}{$0.80(0.25-2.51)$} \\
\hline & & GG & $5(4.42)$ & $8(5.48)$ & & & \\
\hline & \multirow{2}{*}{ allelic model } & A & $190(84.07)$ & $228(78.08)$ & \multirow{2}{*}{2.93} & \multirow{2}{*}{0.08} & \multirow{2}{*}{$1.18(0.94-2.33)$} \\
\hline & & G & $36(15.93)$ & $64(21.92)$ & & & \\
\hline
\end{tabular}

MS: Multiple Sclerosis; CYP24A1 genotypes (CC = wild type; $\mathrm{CT}=$ heterozygote; $\mathrm{CC}=$ mutant type); $\mathrm{CYP} 27 \mathrm{~B} 1$ genotypes $(\mathrm{AA}=$ wild type; $\mathrm{AG}=$ heterozygote; $\mathrm{GG}=$ mutant type).

statistically significant differences in the proportions of either polymorphism were found between the two study groups $(\mathrm{p}=0.50$ for $\mathrm{rs} 2248359$ and $\mathrm{p}=0.18$ for rs703842). However, regarding the dominant genetic and the allelic models, there was a trend for a higher frequency of the minor $G$ allele of rs703842 in controls than patients, but these results remained slightly below significance $(\mathrm{p}=$ 0.07 and $p=0.08$ respectively). These results suggest that both SNPs were not associated with MS and thus did not influence the risk of developing MS disease among Moroccans.

Results of the association tests of each genotyped SNP with clinical characteristics of patients are summarized in Table 3, and with DSLs in patients and controls in Table 4. Neither rs2248359 nor rs703842 had any impact on age at onset, MS type, EDSS score or MSSS within MS patients. Similarly, no association was found between the two SNPs genotypes and DSLs in our series ( $p>0.05$ for all).

\section{Discussion}

Hypovitaminosis D has been proposed as a possible risk factor for MS over 40 
Tables 3. Comparison of MS patients' clinical characteristics between the different genotypes of rs2248359 (CYP24A1) and rs703842 (CYP27B1) polymorphisms.

\begin{tabular}{|c|c|c|c|c|c|c|c|c|c|}
\hline \multirow{2}{*}{$\begin{array}{c}\text { Clinical } \\
\text { characteristics }\end{array}$} & \multicolumn{5}{|c|}{ rs2248359 (CYP24A1) } & \multicolumn{4}{|c|}{ rs703842 (CYP27B1) } \\
\hline & $\mathrm{N}$ & $\begin{array}{c}\text { CC } \\
\mathrm{n}=28\end{array}$ & $\begin{array}{c}\mathrm{CT} \\
\mathrm{n}=59\end{array}$ & $\begin{array}{c}\text { TT } \\
\mathrm{n}=26\end{array}$ & $\mathrm{p}$ & $\begin{array}{c}\text { CC } \\
\mathrm{n}=82\end{array}$ & $\begin{array}{c}\text { CT } \\
\mathrm{n}=26\end{array}$ & $\begin{array}{c}\text { TT } \\
\mathrm{n}=5\end{array}$ & $\mathrm{p}$ \\
\hline Age at onset (years) ${ }^{1}$ & 113 & $30.64 \pm 10.49$ & $29.81 \pm 9.81$ & $28.96 \pm 10.05$ & 0.83 & $30.20 \pm 10.43$ & $29.12 \pm 8.34$ & $27.40 \pm 11.28$ & 0.77 \\
\hline MS type ${ }^{2}:$ & 113 & & & & & & & & \\
\hline RRMS and SPMS & 90 & $20(17.70)$ & 47 (41.59) & $23(20.35)$ & 0.30 & $65(57.52)$ & $21(18.58)$ & $4(3.54)$ & 0.99 \\
\hline PPMS & 23 & $8(7.08)$ & $12(10.62)$ & $3(2.65)$ & & $17(15.04)$ & $5(4.42)$ & $1(0.88)$ & \\
\hline EDSS score $^{1}$ & 113 & $3.75 \pm 2.16$ & $3.39 \pm 1.81$ & $3.37 \pm 1.96$ & 0.68 & $3.35 \pm 1.79$ & $3.86 \pm 2.29$ & $3.40 \pm 2.30$ & 0.50 \\
\hline MSSS $^{1}$ & 113 & $6.03 \pm 3.07$ & $5.56 \pm 2.66$ & $5.10 \pm 2.94$ & 0.49 & $5.52 \pm 2.73$ & $5.95 \pm 3.14$ & $4.46 \pm 2.80$ & 0.53 \\
\hline
\end{tabular}

${ }^{1}$ Mean and standard deviation; ${ }^{2}$ Number and percentage n (\%); MS: Multiple Sclerosis; RRMS: Relapsing-Remitting Multiple Sclerosis; SPMS: Secondary Progressive Multiple Sclerosis; PPMS: Primary Progressive Multiple Sclerosis; EDSS: Expanded Disability Status Scale; MSSS: Multiple Sclerosis Severity Score; $\mathrm{CYP} 24 \mathrm{~A} 1$ genotypes $(\mathrm{CC}=$ wild type; $\mathrm{CT}=$ heterozygote; $\mathrm{CC}=$ mutant type); $\mathrm{CYP} 27 \mathrm{~B} 1$ genotypes $(\mathrm{AA}=$ wild type; $\mathrm{AG}=$ heterozygote; $\mathrm{GG}=$ mutant type).

Table 4. Comparison of vitamin D3 serum levels between the different genotypes of rs2248359 (CYP24A1) and rs703842 (CYP27B1) polymorphisms in patients and controls.

\begin{tabular}{|c|c|c|c|c|c|c|c|c|c|}
\hline & \multirow[b]{2}{*}{$\mathrm{N}$} & \multicolumn{4}{|c|}{ rs2248359 (CYP24A1) } & \multicolumn{4}{|c|}{ rs703842 (CYP27B1) } \\
\hline & & $\begin{array}{c}\text { CC } \\
\mathrm{n}=28\end{array}$ & $\begin{array}{c}\mathrm{CT} \\
\mathrm{n}=59\end{array}$ & $\begin{array}{c}\mathrm{TT} \\
\mathrm{n}=26\end{array}$ & $\mathrm{p}$ & $\begin{array}{c}\text { CC } \\
\mathrm{n}=82\end{array}$ & $\begin{array}{c}\text { CT } \\
\mathrm{n}=26\end{array}$ & $\begin{array}{c}\text { TT } \\
\mathrm{n}=5\end{array}$ & $\mathrm{p}$ \\
\hline \multicolumn{10}{|l|}{$\mathrm{DSL}(\mathrm{ng} / \mathrm{mL})^{1}$ : } \\
\hline MS patients & 113 & $10.30 \pm 5.23$ & $12.40 \pm 7.87$ & $11.62 \pm 6.45$ & 0.42 & $11.54 \pm 6.57$ & $12.07 \pm 7.54$ & $12.40 \pm 11.52$ & 0.92 \\
\hline Controls & 146 & $13.25 \pm 6.90$ & $12.17 \pm 6.17$ & $14.14 \pm 6.92$ & 0.34 & $12.90 \pm 6.35$ & $13.67 \pm 7.22$ & $9.76 \pm 4.65$ & 0.30 \\
\hline
\end{tabular}

${ }^{1}$ Mean and standard deviation; DSL: vitamin D3 serum level; MS: Multiple Sclerosis; CYP24A1 genotypes $(\mathrm{CC}=$ wild type; $\mathrm{CT}=$ heterozygote; $\mathrm{CC}=$ mutant type); CYP27B1 genotypes (AA = wild type; $\mathrm{AG}=$ heterozygote; $\mathrm{GG}=$ mutant type).

years ago, initially to explain the observation of a latitude gradient in MS prevalence [18]. Since then, several studies have focused on the subject to see if low DSLs were associated with a high risk of developing MS, but results obtained are conflicting. Indeed, some case-control studies reported no difference in DSLs between MS patients and controls [19] [20] [21] [22] [23], while others have observed lower DSLs in MS patients [24]-[31]. In Morocco, we did not find an association between DSL and MS status in a previous study; but our ability to detect an association might have been limited by a very high rate (approximately 98\%) of hypovitaminosis D observed in both MS patients and controls (in press).

In parallel, given the possible link of vitamin D with MS susceptibility, it was anticipated that genetic variants of genes involved in DSL regulation might have an influence on MS risk. Studies about the involvement of these genes in MS susceptibility are predominantly focused on association between SNPs and the disease, but surprisingly, most do not relate it to vitamin D concentrations or MS severity markers; Further research is needed in this direction. The most important to date was a collaborative GWAS conducted by the International Multiple Sclerosis Genetics Consortium and the Wellcome Trust Case Control Con- 
sortium 2 (IMSGC/WTCCC2) in 15 different countries, on nearly 10,000 MS patients and 17,000 controls, almost all from European ancestry [13]. This cohort was then supplemented by about 15,000 patients and 25,000 controls [32]. Both SNPs (rs2248359 and rs703842) have been reported to be associated with MS in the first study and after enlargement of the cohort. Against this background, and in an effort to investigate the association reported by the IMSGC/WTCCC2 study [13] within the Moroccan population, we conducted a preliminary assessment of rs2248359 and rs703842 effects on MS risk, some clinical features (age at onset, MS type, EDSS score and MSSS) and DSL.

Our results showed no association between the tested SNPs and MS status. However, since the $\mathrm{p}$ value was very close to the significance threshold when considering the dominant genetic and the allelic models, it should be mentioned that an interesting trend emerges concerning rs703842 (CYP27B1). In fact, the minor $\mathrm{G}$ allele carriers (one or two copies) are more likely to not develop MS than non-carriers. This result suggests that the $\mathrm{G}$ allele might have a protective effect on developing MS in our population. Results from previous studies diverge again. Regarding rs2248359 (CYP24A1), besides the IMSGC/WTCCC2 study [13], only one other found an association between this polymorphism and MS risk. It was conducted exclusively on African Americans subjects (803 MS cases and 1516 controls) in order to determine if MS-associated loci discovered in European populations also influence susceptibility in African Americans [33]. However, two other studies found no association between rs2248359 and MS status within German [34] and Chinese populations [35]. The same applies to rs703842 (CYP27B1), as in the IMSGC/WTCCC2 study [13], it has been identified as a confirmed MS susceptibility locus in another GWAS conducted by the Australia and New Zealand Multiple Sclerosis Genetics Consortium (ANZgene) on 3874 MS cases and 5723 controls of European ancestry [12]. This result has been replicated by two studies which include in their samples subsets of the IMSGC or ANZgene cohorts [36] [37] and two other studies within Swedish [38] and Chinese populations [35]. Negative results concerning such a link between rs703842 and MS status have also been reported within Canadian [39] and American [40] populations. Furthermore, we found no evidence that any of the tested SNPs modified the clinical phenotypes of age at onset, MS type, EDSS score or MSSS in our patients. The same finding was reported by an Australian study for rs703842 (CYP27B1) [41]. We also did not detect an effect of the two SNPs on DSL, although they were expected to modulate it. This was consistent with an Anglo-American study showing no association between rs703842 (CYP27B1) and DSL [37], but contrasted with a Canadian one in which DSL differed significantly between the different CYP27B1 genotypes [39].

The divergence of results obtained from each study is likely due to ethnic differences between the studied populations, but environmental or behavioral factors related to vitamin $\mathrm{D}$ could also influence the expected association between the two SNPs and MS risk [37]. Interestingly, similar studies on association of vitamin D receptor (VDR) polymorphisms with MS or with type 1 diabetes mel- 
litus (another disease that has been associated with DSL through the literature) suggested that an association might only be penetrant in populations with an adequate vitamin D exposure [42] [43] [44]. Hypothetically in a similar way, the $\mathrm{T}$ allele of CYP24A1 and the G allele of CYP27B1 that yield protection for developing MS might not affect disease status in a population generally deficient in vitamin D like ours. Indeed, about $98 \%$ of our control subjects had hypovitaminosis D (in press), which could explain why we did not find an association. Almost all studies related to CYP24A1 and CYP27B1 mentioned above had not measured DSLs, nor even taken into account determinants of circulating vitamin D (sun exposure, shadow-seeking behavior, skin pigmentation, use of sunscreen, wearing concealing clothing, dietary intake, multivitamin or cod-liver oil supplementation...). Therefore, it is possible that those which reported an association involved populations with high exposure to vitamin $\mathrm{D}$.

There are certain limitations to the present study. Indeed, because of the relatively small sample size, we were underpowered to detect modest effects of SNPs on DSL or MS. Therefore, our findings only provide evidence against the existence of strong effects. Then, the case-control approach we used can lead to imperfect matching between cases and controls. Potential associations may be consequently under or over-estimated. A family-based design would have been more appropriate for this kind of study, but unfortunately, relatives were often not available for included patients. Otherwise, we did a single DSL measure for each participant, at the time of recruitment. Thus, DSLs obtained may not reflect the long-term DSL status. Moreover, due to the inclusion of prevalent MS cases, we could not always measure DSLs of patients at disease onset. This would have allowed us to better evaluate the SNPs effects on DSL in patients, before some disease symptoms change their behavioral habits regarding sun exposure.

\section{Conclusion}

In conclusion, there was no convincing evidence that rs2248359 (CYP24A1) and rs703842 (CYP27B1) were associated with MS risk, some clinical features (age at onset, MS type, EDSS score and MSSS) or DSL within the Moroccan population. These findings require further confirmation from large independent studies; and it would also be interesting to carry out similar studies in other populations ethnically close to ours (Algeria, Libya...) to see if there are similarities.

\section{Acknowledgements}

The authors thank all the patients and controls who participate in this study. This work was supported by the National Center for Scientific and Technical Research (CNRST, Morocco) and the Mohammed V University in Rabat (UM5R, Morocco).

\section{References}

[1] Haussleiter, I.S., Brüne, M. and Juckel, G. (2009) Psychopathology in Multiple Sclerosis: Diagnosis, Prevalence and Treatment. Therapeutic Advances in Neurological Disorders, 2, 13-29. https://doi.org/10.1177/1756285608100325 
[2] Stadelmann, C., Wegner, C. and Brück, W. (2011) Inflammation, Demyelination, and Degeneration-Recent Insights from MS Pathology. Biochimica et Biophysica Acta, 1812, 275-282. https://doi.org/10.1016/j.bbadis.2010.07.007

[3] Goodin, D.S. (2009) The Causal Cascade to Multiple Sclerosis: A Model for MS Pathogenesis. PLoS One, 4, e4565. https://doi.org/10.1371/journal.pone.0004565

[4] Oksenberg, J.R. and Baranzini, S.E. (2010) Multiple Sclerosis Genetics-Is the Glass Half Full, or Half Empty? Nature Reviews Neurology, 6, 429-437. https://doi.org/10.1038/nrneurol.2010.91

[5] Smolders, J., Damoiseaux, J., Menheere, P. and Hupperts, R. (2008) Vitamin D as an Immune Modulator in Multiple Sclerosis, a Review. Journal of Neuroimmunology, 194, 7-17. https://doi.org/10.1016/j.jneuroim.2007.11.014

[6] Dörr, J., Döring, A. and Friedemann, P. (2013) Can We Prevent or Treat Multiple Sclerosis by Individualised Vitamin D Supply? The EPMA Journal, 4, 4. https://doi.org/10.1186/1878-5085-4-4

[7] Pierrot-Deseilligny, C. and Souberbielle, J.C. (2013) Contribution of Vitamin D Insufficiency to the Pathogenesis of Multiple Sclerosis. Therapeutic Advances in Neurological Disorders, 6, 81-116. https://doi.org/10.1177/1756285612473513

[8] International Multiple Sclerosis Genetics Consortium (IMSGC) (2007) Risk Alleles for Multiple Sclerosis Identified by a Genomewide Study. The New England Journal of Medicine, 357, 851-862. https://doi.org/10.1056/NEJMoa073493

[9] Wellcome Trust Case Control Consortium (WTCCC) and The Australo-AngloAmerican Spondylitis Consortium (TASC) (2007) Association Scan of 14,500 nsSNPs in Four Common Diseases Identifies Variants Involved in Autoimmunity. Nature Genetics, 39, 1329-1337. https://doi.org/10.1038/ng.2007.17

[10] Baranzini, E.S., Wang, J., Gibson, R.A., Galwey, N., Naegelin, Y., et al. (2009) Genome-Wide Association Analysis of Susceptibility and Clinical Phenotype in Multiple Sclerosis. Human Molecular Genetics, 18, 767-778. https://doi.org/10.1093/hmg/ddn388

[11] De Jager, P.L., Jia, X., Wang, J., de Bakker, P.I.W., Ottoboni, L., et al. (2009) Meta-Analysis of Genome Scans and Replication Identify CD6, IRF8 and TNFRSF1A as New Multiple Sclerosis Susceptibility Loci. Nature Genetics, 41, 776-782. https://doi.org/10.1038/ng.401

[12] The Australia and New Zealand Multiple Sclerosis Genetics Consortium (ANZgene) (2009) Genome-Wide Association Study Identifies New Multiple Sclerosis Susceptibility Loci on Chromosomes 12 and 20. Nature Genetics, 41, 824-828. https://doi.org/10.1038/ng.396

[13] The International Multiple Sclerosis Genetics Consortium (IMSGC) and Wellcome Trust Case Control Consortium 2 (WTCCC2) (2011) Genetic Risk and a Primary Role for Cell-Mediated Immune Mechanisms in Multiple Sclerosis. Nature, 476, 214-219.

[14] Prosser, D. and Jones, G. (2004) Enzymes Involved in The Activation and Inactivation of Vitamin D. Trends in Biochemical Sciences, 29, 664-673.

[15] Ramasamy, A., Trabzuni, D., Forabosco, P., Smith, C., Walker, R., et al. (2014) Genetic Evidence for a Pathogenic Role for the Vitamin D3 Metabolizing Enzyme CYP24A1 in Multiple Sclerosis. Multiple Sclerosis and Related Disorders, 3, 211-219.

[16] Jiang, T., Li, L., Wang, Y., Zhao, C., Yang, J., et al. (2016) The Association between Genetic Polymorphism rs703842 in CYP27B1 and Multiple Sclerosis. Medicine, 95, e3612. https://doi.org/10.1097/MD.0000000000003612

[17] Roxburgh, R.H., Seaman, S.R., Masterman, T., Hensiek, A.E., Sawcer, S.J. and Vu- 
kusic, S. (2005) Multiple Sclerosis Severity Score: Using Disability and Disease Duration to Rate Disease Severity. Neurology, 64, 1144-1151. https://doi.org/10.1212/01.WNL.0000156155.19270.F8

[18] Goldberg, P. (1974) Multiple Sclerosis: Vitamin D and Calcium as Environmental Determinants of Prevalence (a Viewpoint) Part 1: Sunlight, Dietary Factors and Epidemiology. International Journal of Environmental Studies, 6, 19-27. https://doi.org/10.1080/00207237408709630

[19] Soilu-Hänninen, M., Airas, L., Mononen, I., Heikkilä, A., Viljanen, M. and Hänninen, A. (2005) 25-Hydroxyvitamin D Levels in Serum at the Onset of Multiple Sclerosis. Multiple Sclerosis, 11, 266-271. https://doi.org/10.1191/1352458505ms1157oa

[20] Van der Mei, I.A., Ponsonby, A.L., Dwyer, T., Blizzard, L., Taylor, B.V., et al. (2007) Vitamin D Levels in People with Multiple Sclerosis and Community Controls in Tasmania, Australia. Journal of Neurology, 254, 581-590. https://doi.org/10.1007/s00415-006-0315-8

[21] Barnes, M.S., Bonham, M.P., Robson, P.J., Strain, J.J., Lowe-Strong, A.S., Eaton-Evans, J., et al. (2007) Assessment of 25-Hydroxyvitamin D and 1,25-Dihydroxyvitamin D3 Concentrations in Male and Female Multiple Sclerosis Patients and Control Volunteers. Multiple Sclerosis, 13, 670-672. https://doi.org/10.1177/1352458506072666

[22] Lonergan, R., Kinsella, K., Fitzpatrick, P., Brady, J., Murray, B., Dunne, C., et al. (2011) Multiple Sclerosis Prevalence in Ireland: Relationship to Vitamin D Status and HLA Genotype. Journal of Neurology, Neurosurgery, and Psychiatry, 82, 317-322. https://doi.org/10.1136/jnnp.2010.220988

[23] Moen, S.M., Celius, E.G., Sandvik, L., Brustad, M., Nordsletten, L., Eriksen, E.F. and Holmøy, T. (2012) Bone Turnover and Metabolism in Patients with Early Multiple Sclerosis and Prevalent Bone Mass Deficit: A Population-Based Case-Control Study. PLoS ONE, 7, e45703. https://doi.org/10.1371/journal.pone.0045703

[24] Ozgocmen, S., Bulut, S., Ilhan, N., Gulkesen, A., Ardicoglu, O. and Ozkan, Y. (2005) Vitamin D Deficiency and Reduced Bone Mineral Density in Multiple Sclerosis: Effect of Ambulatory Status and Functional Capacity. Journal of Bone and Mineral Metabolism, 23, 309-313. https://doi.org/10.1007/s00774-005-0604-9

[25] Gelfand, J.M., Cree, B.A.C., McElroy, J., Oksenberg, J., Green, R., et al. (2011) Vitamin D in African Americans with Multiple Sclerosis. Neurology, 76, 1824-1830. https://doi.org/10.1212/WNL.0b013e31821cccf5

[26] Neau, J.P., Artaud-Uriot, M.S., Lhomme, V., Bounaud, J.Y., Lebras, F., Boissonnot, L., et al. (2011) Vitamine D et Sclérose en Plaques. Etude Prospective d'une Cohorte de Patients de la Région Poitou-Charentes. [Vitamin D and Multiple Sclerosis. A Prospective Survey of Patients of Poitou-Charentes Area.] Revue Neurologique, 167, 317-323.

[27] Bäärnhielm, M., Hedström, A.K., Kockum, I., Sundqvist, E., Gustafsson, S.A., et al. (2012) Sunlight Is Associated with Decreased Multiple Sclerosis Risk: No Interaction with Human Leukocyte Antigen-DRB1 15. European Journal of Neurology, 19, 955-962. https://doi.org/10.1111/j.1468-1331.2011.03650.x

[28] Mouhieddine, T.H., Darwish, H., Fawaz, L., Yamout, B., Tamim, H. and Khoury, S.J. (2015) Risk Factors for Multiple Sclerosis and Associations with Anti-EBV Antibody Titers. Clinical Immunology, 158, 59-66.

[29] Niino, M., Sato, S., Fukazawa, T., Masaki, K., Miyazaki, Y., et al. (2015) Decreased Serum Vitamin D Levels in Japanese Patients with Multiple Sclerosis. Journal of Neuroimmunology, 279, 40-45.

[30] Karampoor, S., Zahednasab, H., Ramagopalan, S., Mehrpour, M., Safarnejad Ta- 
meshkel, F. and Keyvani, H. (2016) 25-Hydroxyvitamin D Levels Are Associated with Multiple Sclerosis in Iran: A Cross-Sectional Study. Journal of Neuroimmunology, 290, 47-48.

[31] Saeedan, M., Ko, Y.G., Chirakkara, S.K.P., Sinha, S. and Shatila, A. (2017) Hypovitaminosis D Status in Newly Diagnosed Cases of MS versus Control Group. Journal of Multiple Sclerosis, 4, 199. https://doi.org/10.4172/2376-0389.1000199

[32] International Multiple Sclerosis Genetics Consortium (IMSGC), Wellcome Trust Case Control Consortium 2 (WTCCC2) and International IBD Genetics Consortium (IIBDGC) (2013) Analysis of Immune-Related Loci Identifies 48 New Susceptibility Variants for Multiple Sclerosis. Nature Genetics, 45, 1353-1360.

https://doi.org/10.1038/ng.2770

[33] Isobe, N., Madireddy, L., Khankhanian, P., Matsushita, T., Caillier, S.J., et al. (2015) An ImmunoChip Study of Multiple Sclerosis Risk in African Americans. Brain, 138, 1518-1530. https://doi.org/10.1093/brain/awv078

[34] Akkad, D.A., Olischewsky, A., Reiner, F., Hellwig, K., Esser, S., Epplen, J.T., et al. (2015) Combinations of Susceptibility Genes Are Associated with Higher Risk for Multiple Sclerosis and Imply Disease Course Specificity. PLoS ONE, 10, e0127632. https://doi.org/10.1371/journal.pone.0127632

[35] Zhuang, J.C., Huanga, Z.Y., Zhao, G.X., Yua, H., Li, Z.X. and Wua, Z.Y. (2015) Variants of CYP27B1 Are Associated with Both Multiple Sclerosis and Neuromyelitis Optica Patients in Han Chinese Population. Gene, 557, 236-239.

[36] Cortes, A., Field, J., Glazov, E.A., Hadler, J., ANZgene Consortium, Stankovich, J. and Brown, M.A. (2013) Resequencing and Fine-Mapping of the Chromosome 12q13-14 Locus Associated with Multiple Sclerosis Refines the Number of Implicated Genes. Human Molecular Genetics, 22, 2283-2292. https://doi.org/10.1093/hmg/ddt062

[37] Simon, K.C., Munger, K.L., Kraft, P., Hunter, D.J., De Jager, P.L. and Ascherio, A. (2011) Genetic Predictors of 25-Hydroxyvitamin D Levels and Risk of Multiple Sclerosis. Journal of Neurology, 258, 1676-1682. https://doi.org/10.1007/s00415-011-6001-5

[38] Sundqvist, E., Bäärnhielm, M., Alfredsson, L., Hillert, J., Olsson, T. and Kockum, I. (2010) Confirmation of Association between Multiple Sclerosis and CYP27B1. European Journal of Human Genetics, 18, 1349-1352. https://doi.org/10.1038/ejhg.2010.113

[39] Orton, S.M., Morris, A.P., Herrera, B.M., Ramagopalan, S.V., Lincoln, M.R., Chao, M.J., et al. (2008) Evidence for Genetic Regulation of Vitamin D Status in Twins with Multiple Sclerosis. The American Journal of Clinical Nutrition, 88, 441-447.

[40] Simon, K.C., Munger, K.L., Yang, X. and Ascherio, A. (2010) Polymorphisms in Vitamin D Metabolism Related Genes and Risk of Multiple Sclerosis. Multiple Sclerosis, 16, 133. https://doi.org/10.1177/1352458509355069

[41] Jensen, C.J., Stankovich, J., Van der Walt, A., Bahlo, M., Taylor, B.V., van der Mei, I.A.F., et al. (2010) Multiple Sclerosis Susceptibility-Associated SNPs Do Not Influence Disease Severity Measures in a Cohort of Australian MS Patients. PLoS ONE, 5, e10003. https://doi.org/10.1371/journal.pone.0010003

[42] Smolders, J., Damoiseaux, J., Menheere, P., Tervaert, J.W. and Hupperts, R. (2009) Fokl Vitamin D Receptor Gene Polymorphism (rs10735810) and Vitamin D Metabolism in Multiple Sclerosis. Journal of Neuroimmunology, 207, 117-121.

[43] Smolders, J., Damoiseaux, J., Menheere, P., Tervaert, J.W. and Hupperts, R. (2009) Association Study on Two Vitamin D Receptor Gene Polymorphisms and Vitamin 
D Metabolites in Multiple Sclerosis. Annals of the New York Academy of Sciences, 1173, 515-520. https://doi.org/10.1111/j.1749-6632.2009.04656.x

[44] Ponsonby, A.L., Pezic, A., Ellis, J., Morley, R., Cameron, F., Carlin, J. and Dwyer, T. (2008) Variation in Associations between Allelic Variants of the Vitamin D Receptor Gene and Onset of Type 1 Diabetes Mellitus by Ambient Winter Ultraviolet Radiation Levels: A Meta-Regression Analysis. American Journal of Epidemiology, 168, 358-365. https://doi.org/10.1093/aje/kwn142 